\title{
Autonomic dysfunction predicts poor outcome in stroke: Updates on recent autonomic research
}

\author{
Mitchell G. Miglis ${ }^{1} \cdot$ Srikanth Muppidi ${ }^{1}$
}

Received: 20 December 2017 / Accepted: 21 December 2017 / Published online: 5 January 2018

(c) Springer-Verlag GmbH Germany, part of Springer Nature 2018

Keywords Alpha-synucleinopathy $\cdot$ Pure autonomic failure $\cdot$ Sympathetic denervation · Orthostatic hypotension $\cdot$ Stroke $\cdot$ Dysautonomia

\section{Autonomic dysfunction predicts negative outcome after ischemic stroke}

Inpatient neurologists are well aware of how common autonomic dysfunction is in patients with acute stroke. For those of us who no longer practice inpatient medicine, we can still remember the many stroke patients we cared for during our neurology training: labile blood pressures, refractory hypertension, fevers, and cardiac arrhythmias were not uncommon, despite aggressive treatments. Research has demonstrated that impaired heart rate variability correlates with both post-stroke disability [4] and all-cause mortality [5]. What is less clear is the how the magnitude of autonomic dysfunction in the acute stroke period influences stroke recovery.

Xiong and colleagues have attempted to help answer this question in their recent paper published in the December 4, 2017 issue of Stroke [6]. The authors recruited 150 adult patients who were admitted for mild acute ischemic stroke (NIHSS 4-10). Stroke was confirmed on imaging, and patients were recruited within 7 days of stroke symptom onset. Patients with arrhythmias such as atrial fibrillation were excluded, as were those with hemodynamic instability or major concurrent illness (ex. chronic obstructive pulmonary disease, renal failure, active malignancy), as these variables may have altered autonomic testing results.

Autonomic cardiovascular testing was performed with a portable system (Task Force Monitoring, CNSystems) capable of measuring beat-to-beat BP and HR. All patients underwent measures of HR variability with deep breathing,

Srikanth Muppidi

muppidis@stanford.edu

1 Department of Neurology, Stanford Medical Center, Palo Alto, CA, USA
Valsalva maneuver, sustained handgrip testing, and active standing to calculate BP responses and the 30:15 ratio. Patients were grouped according to the Ewing classification [7]: Normal: all tests normal or one borderline; early involvement: one of the three HR tests abnormal or two borderline; definite involvement: two or more of the HR tests abnormal; severe involvement: two or more of the HR tests abnormal plus one or both of the BP pressure tests abnormal, or both borderline; atypical pattern: any other combination of abnormal tests. Patients with normal or early impairment were classified as "minor autonomic function impairment" by the authors, whereas the remaining patients in the definite, severe, or atypical groups were classified as "significant autonomic function impairment." Patients were reassessed at 3 months, and their outcome was dichotomized based on their Rankin score: good (modified Rankin Scale score 0-2) or poor (modified Rankin Scale score 3-6). The authors then calculated odds ratios of the magnitude of autonomic dysfunction related to poor functional outcome.

Most patients were men (71\%), with a mean age of $66.4 \pm 9.9$ years. The majority $(76.0 \%)$ of patients were classified as having significant autonomic dysfunction. After adjusting for statistically significant confounding variables, such as gender and NIHSS on admission, the magnitude of autonomic dysfunction independently predicted an unfavorable outcome, with an odds ratio of 3.263 (95\% confidence interval, $1.141-9.335 ; p=0.027)$. A poor functional outcome was seen in $32.5 \%$ of patients with significant autonomic impairment, compared to $13.9 \%$ of patients with minor autonomic impairment $(p=0.031)$. Patients in the poor outcome group were more likely to be women, have a higher NIHSS score on admission, and have more significant autonomic dysfunction.

The authors suggest several possible explanations for the greater risk of poor outcome in those with significant 
autonomic impairment. Cerebrovascular autoregulation is impaired in acute ischemic stroke, and an inability to regulate labile blood pressure may result in a greater degree of cerebral ischemia. Other studies have demonstrated a reduction in parasympathetic tone after ischemic stroke [8], and a shift to sympathetic predominance has been shown to increase proinflammatory cytokine production, hyperglycemia, and blood-brain barrier permeability, which would negatively affect stroke recovery. Sympathetic predominance also increases the risk of atrial fibrillation, which impairs recovery.

There are, of course, several limitations to this study. The authors admit that medications such as anti-hypertensives may have affected the test results, as well as the inclusion of diabetic patients, in whom comorbid autonomic dysfunction is common. The autonomic testing was performed in a hospital and not in the controlled environment of an autonomic laboratory, therefore myriad environmental confounders could have influenced test results (food ingestion, hydration status, time of day, sleep deprivation, etc.). The authors did not describe the specific abnormalities seen on autonomic testing, but rather reported the Ewing classification only, which is helpful for statistical analysis but does not add to the understanding of the pathophysiology. It would have been important for the authors to describe the specific components of autonomic testing that were affected, which branches of the autonomic system were more heavily involved (sympathetic vs. parasympathetic), and how these details factor into stroke recovery prognosis. Nevertheless, this study provides objective evidence of what neurologists have anecdotally observed since they began monitoring stroke patients in the hospital setting: acute stroke produces autonomic dysfunction, and further understanding and treatment of this autonomic dysfunction may lead to improved outcomes in stroke survivors.

\section{Getting closer to the truth in pure autonomic failure}

Pure autonomic failure (PAF) is the only $\alpha$-synucleinopathy without significant CNS findings of motor or cognitive impairment, differentiating it from the CNS $\alpha$-synucleinopathies of Parkinson's disease (PD), multiple system atrophy (MSA), and dementia with Lewy bodies (DLB). Longitudinal studies suggest that some patients with PAF may eventually evolve into a central $\alpha$-synucleinopathy over time [1]. In most patients with PAF, autonomic failure is due to $\alpha$-synuclein-mediated post-ganglionic sympathetic denervation. This is in contrast to MSA, in which $\alpha$-synuclein-mediated pathology is typically pre-ganglionic. Recently, investigators have utilized epidermal skin biopsy to observe this pathological change as a potential biomarker in DLB and PD [2]. Other tests such as plasma norepinephrine levels and cardiac sympathetic imaging may aid in the diagnosis; however, few centers routinely perform these tests and they are rarely employed in clinical practice.

While we have clear scientific evidence for peripheral sympathetic impairment in PAF, it is unclear if this sympathetic impairment is functional or structural. To address these questions, Guo and colleagues [3] undertook an elegant study with multimodal investigation of sympathetic function in patients with PAF, MSA and healthy controls. All subjects underwent tilt-table testing with assessment of arterial norepinephrine levels and clearance. In addition, patients had muscle sympathetic nerve activity measured. To measure peripheral sympathetic nerve function, patients had forearm vein biopsies under local anesthesia. Western blot analysis of various norepinephrine-related proteins found in sympathetic nerves was performed on this tissue.

Patients with PAF had normal norepinephrine transporter (NET) levels; however, they had reduced levels of tyrosine hydroxylase $(p<0.0001)$ and vesicular monoamine transporter 2 (VMAT2) protein expression $(p<0.05)$, compared to controls and patients with MSA. In addition, the plasma 3,4-dihydroxyphenylglycol (3,4-DHPG): norepinephrine (NE) ratio was higher in patients with PAF compared to controls $(p<0.05)$. The authors concluded that normal NET levels suggest an intact sympathetic nerve with reduced NE synthesis in patients with PAF. Elevated plasma ratios of 3,4-dihydroxyphenylglycol: norepinephrine and reduced VMAT2 in PAF patients indicate a shift towards intraneuronal norepinephrine metabolism over sequestration in sympathetic nerves. Overall, these findings indicate that there is a likely a sympathetic dysfunction preceding sympathetic nerve degeneration.

This study brings us closer to the etiology of sympathetic and autonomic failure in PAF but has several limitations. First, the sample size was small. Second, staining for peripheral $\alpha$-synuclein would have made it possible to correlate the degree of sympathetic changes and $\alpha$-synuclein deposition, especially since previous work suggests that $\alpha$-synuclein deposition correlates with autonomic failure on testing. As the authors discuss, it is possible that there is differential involvement of sympathetic fibers in different organs, and previous studies documenting $\alpha$-synuclein deposition in epidermal nerve fibers on skin biopsy samples do not correlate with the findings seen in this study. A longitudinal study in a PAF cohort with additional staining methods will help us evaluate this further. Nevertheless, we are getting closer to the truth, and hopefully our improved understanding will lead to therapeutics that can prevent progression of disease. 


\section{Compliance with ethical standards}

Conflict of interests The authors declare that they have no conflict of interest.

\section{References}

1. Kaufmann H, Norcliffe-Kaufmann L, Palma JA et al (2017) Natural history of pure autonomic failure: a United States prospective cohort. Ann Neurol 81(2):287-297

2. Wang N, Gibbons CH, Lafo J, Freeman R (2013) $\alpha$-Synuclein in cutaneous autonomic nerves. Neurology 81(18):1604-1610. http s://doi.org/10.1212/wnl.0b013e3182a9f449

3. Guo L, Esler MD, Sari C et al (2017) Does sympathetic dysfunction occur before denervation in pure autonomic failure? Clin Sci (Lond). https://doi.org/10.1042/cs20170240
4. Korpelainen JT, Sotaniemi KA, Huikuri HV, Myllyä VV (1996) Abnormal heart rate variability as a manifestation of autonomic dysfunction in hemispheric brain infarction. Stroke 27:2059-2063

5. Colivicchi F, Bassi A, Santini M, Caltagirone C (2004) Cardiac autonomic derangement and arrhythmias in right-sided stroke with insular involvement. Stroke 35:2094-2098. https://doi. org/10.1161/01.STR.0000138452.81003.4c

6. Xiong L, Tian G, Leung $\mathrm{H}$ et al (2017) Autonomic dysfunction predicts clinical outcomes after acute ischemic stroke. Stroke. http s://doi.org/10.1161/strokeaha.117.019312

7. Ewing DJ, Borsey DQ, Bellavere F, Clarke BF (1981) Cardiac autonomic neuropathy in diabetes: comparison of measures of R-R interval variation. Diabetologia 21:18-24

8. Dutsch M, Burger M, Dorfler C et al (2007) Cardiovascular autonomic function in poststroke patients. Neurology 69:2249-2255. https://doi.org/10.1212/01.wnl.0000286946.06639.a7 